\title{
Pattern recognition with wavelength-multiplexed filters
}

\author{
S. K. Case
}

\begin{abstract}
Wavelength-multiplexed matched spatial filters are used in an incoherent optical pattern recognition system. The system produces color-coded correlation responses which allow the simultaneous recognition of several objects and, in addition, reduces the requirements on filter positioning devices. Analysis of the system, comparison with previous systems, experimental results, and discussions are given.
\end{abstract}

\section{Introduction}

There is growing interest in temporally incoherent optical processes for such uses as pseudocoloring of images, ${ }^{1}$ to aid a human observer, or wavelength.coding of images for transmission through a low spatial bandpass optical system such as an optical fiber. ${ }^{2,3}$ In this paper, we show that wavelength coding can also be useful for optical pattern recognition systems. Both reflection and transmission wavelength-coded matched spatial filter systems are described. Reflection systems are conceptually the most simple and allow the largest number of multiplexed filters because of their narrow wavelength selectivity. Transmission systems are easier to construct experimentally, especially if fixed wavelength laser readout is used. An analysis and experimental results are given for a transmission colorcoded recognition system.

\section{A. Coherent Pattern Recognition}

Spatially and temporally coherent optical pattern recognition systems have been successfully used to recognize a certain object while discriminating against others. ${ }^{4,5}$ If a number of different objects have to be identified, however, several matched spatial filters (one for each object) are typically recorded next to each other on one holographic plate, and then each filter is sequentially positioned in the Fourier plane for use in the pattern recognition operation. This sequential filter arrangement requires a filter positioning device which affords both great positioning precision, for each filter, and a large distance of travel between filters. It also allows only serial recognition operations.

The author was with Virginia Polytechnic Institute and State University, Physics Department, when this work was performed. $\mathrm{He}$ is currently with the University of Minnesota, Electrical Engineering Department, Minneapolis, Minnesota 55455.

Received 8 August 1978

0003-6935/79/121890-05\$00.50/0

(C) 1979 Optical Society of America.
The above problems can be avoided by using wavelength multiplexing for the series of filters. That is, two or more filters can be spatially superimposed but recorded at different wavelengths. This arrangement reduces the need for expensive filter positioners ${ }^{6}$ and also allows multispectral parallel image processing operations.

\section{B. Color-Coded Pattern Recognition}

As an example of wavelength-multiplexed pattern recognition, consider the use of a double-exposure reflection matched spatial filter (MSF) constructed as follows. A transparency containing the letters GO is first centered in the input plane of an optical processor and illuminated with a coherent plane wave of green light such as that from an argon laser. A lens forms the Fourier transform (FT) of the object which interferes with a reference plane wave to form a reflection MSF. A transparency of the letters STOP is now centered in the input plane, and a second MSF is superimposed on the first using coherent red light such as that from a $\mathrm{He}-\mathrm{Ne}$ laser. After development, the white light impulse response of this filter consists of the word GO in green letters superimposed on the word STOP in red letters.

For pattern recognition, if the GO mask is centered in the input plane, as in Fig. 1(a), and illuminated with spatially coherent, collimated white light, a multispectral FT of the word GO will be incident on the MSF. Only the green portion of the spectrum will be spatially and spectrally matched to the MSF and will be selectively reflected with high efficiency. Thus, the autocorrelation signal obtained after the final FT lens will have an intense green peak at the center of the correlation plane, as shown in Fig. 1(a). The cross correlation between the red portion of the GO spectrum and the STOP matched spatial filter will yield a lower intensity red signal which will also be centered in the correlation plane. In a similar manner, inserting the 


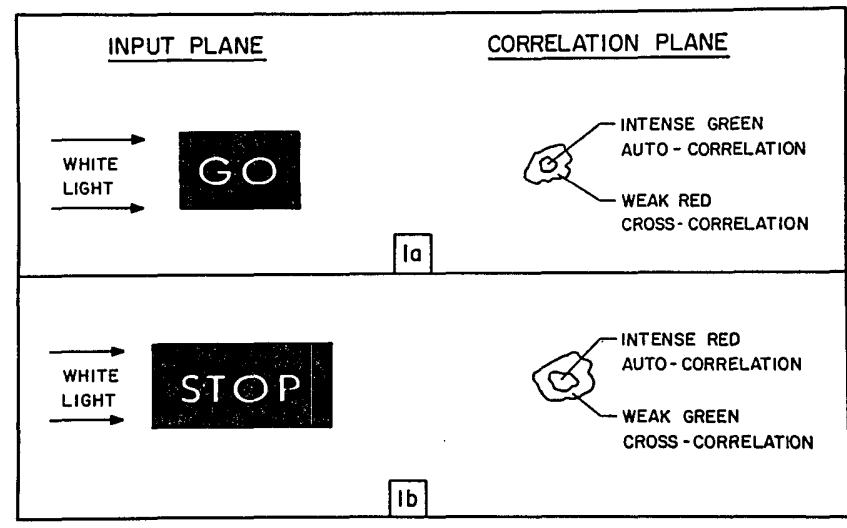

Fig. 1. Color-coded correlation signals: intense green signal is response to GO mask in (a); intense red signal is response to STOP mask in (b). length $\lambda_{1}$ so that the object is $\mathbf{O}\left(x_{1}+x_{0}, y_{1}, \lambda_{1}\right)$. With a Fourier transform (FT) lens of focal length $f_{1}$ and a plane reference wave of amplitude $R$ incident onto the Fourier plane at angle $\theta$ in the $x z$ plane, the Fourier plane amplitude for the first exposure is

$$
\begin{aligned}
U_{21}\left(x_{2}, y_{2}\right)= & R \exp ^{\left.\mid-j\left[\left(2 \pi \sin \theta x_{2}\right) / \lambda_{1}\right]\right\}} \\
& +\frac{1}{j \lambda_{1} f_{1}} \exp ^{\left.\left\{+j\left[\left(2 \pi x_{2} x_{0}\right) / \lambda_{1} f_{1}\right)\right]\right\}} \tilde{\mathbf{O}}\left(\frac{x_{2}}{\lambda_{1} f_{1}}, \frac{y_{2}}{\lambda_{1} f_{1}}\right),
\end{aligned}
$$

where $\tilde{\mathbf{O}}$ stands for the FT of $\mathbf{O}$. The second exposure is made with the object $\mathbf{X}$ centered on the optical axis of the input plane and illuminated with wavelength $\lambda_{2}$, so that the object is $\mathbf{X}\left(x_{1}, y_{1}, \lambda_{2}\right)$. The amplitude in the Fourier plane for the second exposure is

$$
U_{22}\left(x_{2}, y_{2}\right)=R \exp ^{\left.\left\{-j\left[\left(2 \pi \sin \theta x_{2}\right) / \lambda_{2}\right)\right]\right\}}+\frac{1}{j \lambda_{2} f_{1}} \tilde{\mathbf{X}}\left(\frac{x_{2}}{\lambda_{2} f_{1}}, \frac{y_{2}}{\lambda_{2} f_{1}}\right)
$$

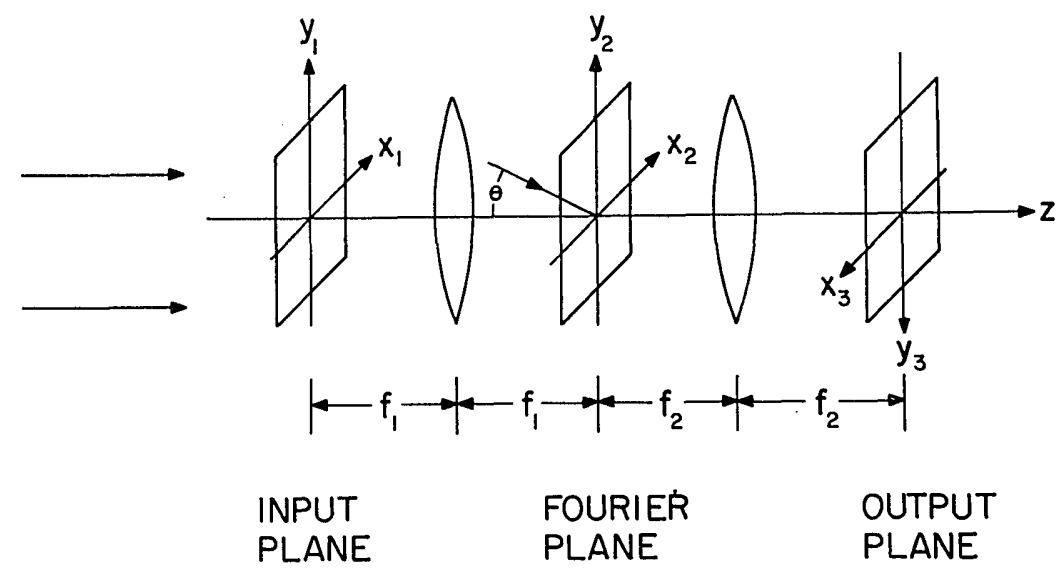

Fig. 2. Optical system.

STOP mask in the input plane will yield an intense red autocorrelation signal and a weak green cross-correlation signal as in Fig. 1(b). This correlation process is also illustrated on the issue cover.

With this multiple exposure MSF, a simultaneous (parallel) search for either of the two input objects can be carried out. A single wavelength discriminating detector, such as a color vidicon, can be placed at the center of the output plane to detect which (if either) of the objects is present at the input. In addition, the color of the correlation response can act as a visual cue to a human observer as in this case with the green-GO and red-STOP signals. ${ }^{14}$

\section{Analysis}

The important question for this parallel processing scheme regards the relative intensities of the superimposed auto-correlation and cross-correlation signals. To test this, a general transmission MSF system is constructed as in Fig. 2. The two objects chosen for these experiments are transparencies of the letters $\mathbf{O}$ and $\mathbf{X}$, to be recorded at wavelengths $\lambda_{1}$ and $\lambda_{2}$, respectively. The object $\mathbf{O}$ is centered at the coordinate $\left(-x_{0}, 0\right)$ in the input plane and illuminated with wave-
The transmittance $t$ of the resulting double-exposure MSF is proportional to $\left|U_{21}\right|^{2}+\left|U_{22}\right|^{2}$. Neglecting all except the two terms which cause diffraction into the correlation plane, we write

$$
\begin{aligned}
t \sim & \frac{1}{\lambda_{1} f_{1}} \exp ^{\left[-j 2 \pi\left(\sin \theta / \lambda_{1}+x_{0} / \lambda_{1} f_{1}\right) x_{2}\right]} \tilde{\mathbf{O}}^{*}\left(\frac{x_{2}}{\lambda_{1} f_{1}}, \frac{y_{2}}{\lambda_{1} f_{1}}\right) \\
& +\frac{1}{\lambda_{2} f_{1}} \exp ^{\left.\left[-j 2 \pi(\sin \theta) / \lambda_{2}\right) x_{2}\right]} \tilde{\mathbf{X}}^{*}\left(\frac{x_{2}}{\lambda_{2} f_{1}}, \frac{y_{2}}{\lambda_{2} f_{1}}\right),
\end{aligned}
$$

where the asterisk indicates the complex conjugate.

For readout, we assume that the test object $\mathbf{O}$ is centered on the optical axis in the input plane and illuminated with polychromatic (temporally incoherent) light, which for the present time is restricted to the two construction wavelengths $\lambda_{2}$ and $\lambda_{1}$. The input signal is

$$
U_{1}=\mathbf{O}\left(x_{1}, y_{1}, \lambda_{2}\right)+\mathbf{O}\left(x_{1}, y_{1}, \lambda_{1}\right)
$$

and the field incident on the MSF is

$$
U_{2}=\frac{1}{j \lambda_{2} f_{1}} \tilde{\mathbf{o}}\left(\frac{x_{2}}{\lambda_{2} f_{1}}, \frac{y_{2}}{\lambda_{2} f_{1}}\right)+\frac{1}{j \lambda_{1} f_{1}} \tilde{\mathbf{o}}\left(\frac{x_{2}}{\lambda_{1} f_{1}}, \frac{y_{2}}{\lambda_{1} f_{1}}\right) .
$$

The amplitude just after the MSF is given by the product of Eqs. (3) and (5)

$$
U_{2+}=t U_{2}
$$




\begin{tabular}{|c|c|c|c|}
\hline$\underline{T e r m}$ & & Amp1itude & Location \\
\hline $\mathrm{U}_{31}\left(\mathrm{x}_{3}, \mathrm{y}_{3}, \lambda_{2}\right)$ & $\frac{\lambda_{1} \mathrm{f}_{1}^{2}}{\lambda_{2} \mathrm{f}_{2}^{2}}$ & {$\left[0\left(\left(x_{3}-x_{31}\right) \frac{\lambda_{1} f_{1}}{\lambda_{2} f_{2}}, y_{3} \frac{\lambda_{1} f_{1}}{\lambda_{2} f_{2}}\right) * 0\left(\left(x_{3}-x_{31}\right) \frac{f_{1}}{f_{2}}, y_{3} \frac{f_{1}}{f_{2}}\right)\right]$} & $x_{31}=\frac{\lambda_{2}}{\lambda_{1}} f_{2} \sin \theta+x_{0} \frac{\lambda_{2}}{\lambda_{1}} \frac{f_{2}}{f_{1}}$ \\
\hline $\mathrm{U}_{32}\left(\mathrm{x}_{3}, \mathrm{y}_{3}, \lambda_{2}\right)$ & $\frac{f_{1}^{2}}{f_{2}^{2}}$ & {$\left[x\left(\left(x_{3}-x_{32}\right) \frac{f_{1}}{f_{2}}, y_{3} \frac{f_{1}}{f_{2}}\right) * 0\left(\left(x_{3}-x_{32}\right) \frac{f_{1}}{f_{2}}, y_{3} \frac{f_{1}}{f_{2}}\right)\right]$} & $x_{32}=f_{2} \sin \theta$ \\
\hline $\mathrm{U}_{33}\left(\mathrm{x}_{3}, \mathrm{y}_{3}, \lambda_{1}\right)$ & $\frac{\mathrm{f}_{1}{ }^{2}}{\mathrm{f}_{2}^{2}}$ & {$\left[0\left(\left(x_{3}-x_{33}\right) \frac{f_{1}}{f_{2}}, y_{3} \frac{f_{1}}{f_{2}}\right) * 0\left(\left(x_{3}-x_{33}\right) \frac{f_{1}}{f_{2}}, y_{3} \frac{f_{1}}{f_{2}}\right)\right]$} & $x_{33}=f_{2} \sin \theta+x_{0} \frac{f_{2}}{f_{1}}$ \\
\hline $\mathrm{U}_{34}\left(\mathrm{x}_{3}, \mathrm{y}_{3}, \lambda_{1}\right)$ & $\frac{\lambda_{2} \mathrm{f}_{1}^{2}}{\lambda_{1} \mathrm{f}_{2}^{2}}$ & {$\left[x\left(\left(x_{3}-x_{34}\right) \frac{\lambda_{2} f_{1}}{\lambda_{1} f_{2}}, y_{3} \frac{\lambda_{2} f_{1}}{\lambda_{1} f_{2}}\right) * 0\left(\left(x_{3}-x_{34}\right) \frac{f_{1}}{f_{2}}, y_{3} \frac{f_{1}}{f_{2}}\right)\right]$} & $x_{34}=\frac{\lambda_{1}}{\lambda_{2}} f_{2} \sin \theta$ \\
\hline
\end{tabular}

The output field $U_{3}$ (FT of $U_{2+}$ ) contains four terms listed in Table I where the symbol $\star$ stands for correlation.

The first and fourth terms represent cross correlations of the input object $\mathbf{O}$ with the size-altered objects $\mathbf{O}$ and $\mathbf{X}$, respectively. The two middle terms are of greater interest for they represent the cross correlation of $\mathbf{X}$ and $\mathbf{O}$, at wavelength $\lambda_{2}$, and the autocorrelation of input $\mathbf{O}$, at wavelength $\lambda_{1}$. Note that if the MSF had been constructed with both objects centered on the optical axis $\left(x_{0}=0\right)$, as described in Sec. I, signals $U_{32}$ and $U_{33}$ would be superimposed and could be detected with a single wavelength-discriminating detector. For this test we chose to physically separate the signals to show their individual shapes more clearly. Constructing the MSF such that term $U_{33}$ is centered between $U_{32}$ and $U_{34}$ requires

$$
x_{0}=\frac{1}{2}\left(\frac{\lambda_{1}}{\lambda_{2}}-1\right) f_{1} \sin \theta .
$$

The correlation terms such as $U_{33}$ and $U_{34}$ are then physically separated ${ }^{7}$ for input object widths $W_{0}=W_{x}$ $=W$ if

$$
W \leq \frac{\left(\frac{\lambda_{1}}{\lambda_{2}}-1\right) f_{1} \sin \theta}{\left(\frac{\lambda_{1}}{\lambda_{2}}+3\right)} .
$$

If we assume Eq. (7) to be true, and $f_{1}=f_{2}=f$, we can write the output in simplified notation as in Table II. Each correlation signal listed in the third column of Table II is written in terms of relative coordinates in the output plane, while the center location of each signal is
Table II. Correlation Plane Output with $\mathrm{O}$ Input

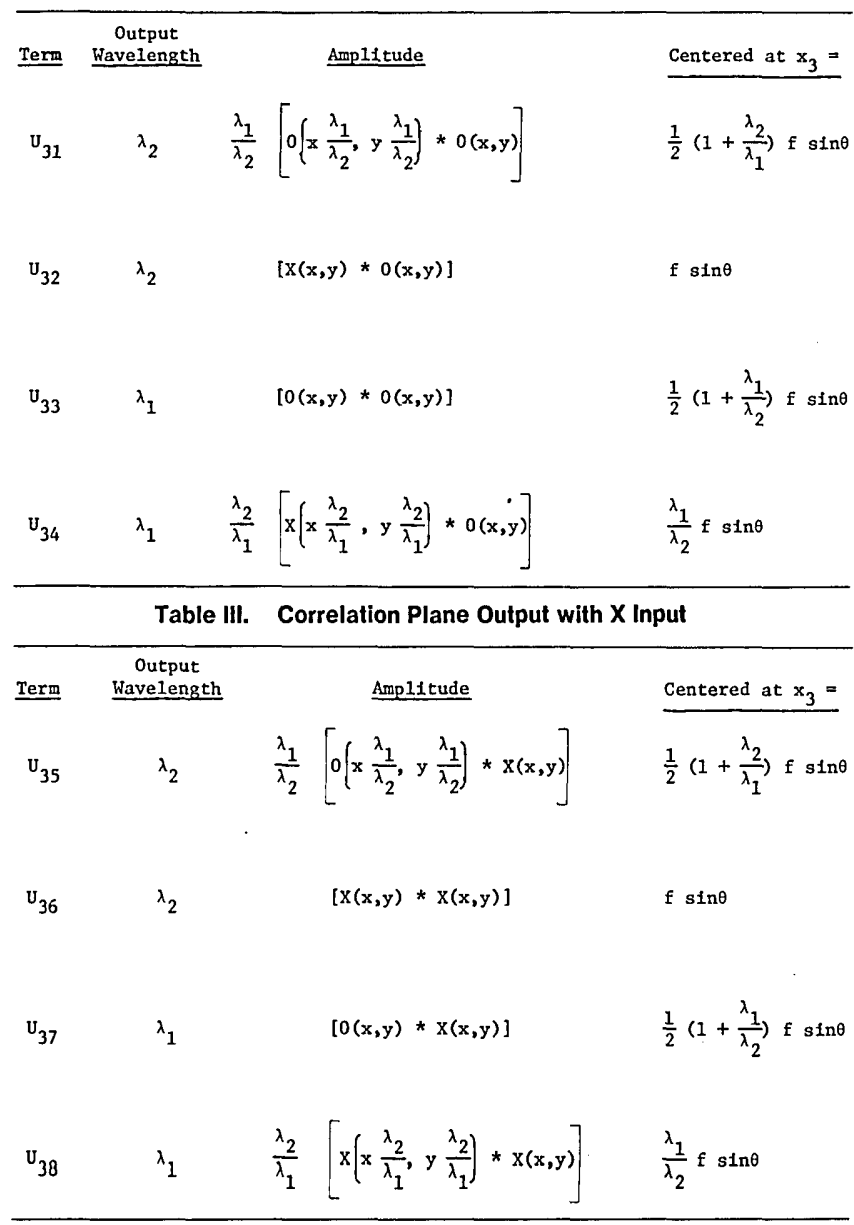


listed in the fourth column. If the transparency of the letter $\mathbf{X}$ is placed at the input of the processor, the output is as indicated in. Table III.

\section{Experimental Results}

The experiments were carried out with $\lambda_{1}=5145 \AA$, $\lambda_{2}=4880 \AA, \theta=9.7^{\circ}, f_{1}=620 \mathrm{~mm}, x_{0}=2.8 \mathrm{~mm}$, and $W$ $=1 \mathrm{~mm}$. The MSF was constructed on Kodak $649-\mathrm{F}$ film. The input transparencies were recorded on Kodalith film and inserted in a liquid gate in the input plane.

The impulse response of the double-exposure MSF when illuminated with wavelengths $\lambda_{1}$ and $\lambda_{2}$ is shown in Fig. 3. From left to right the signals are

$$
\mathbf{O}\left(x \frac{\lambda_{1}}{\lambda_{2}}, y \frac{\lambda_{1}}{\lambda_{2}}, \lambda_{2}\right), \mathbf{X}\left(x, y, \lambda_{2}\right), \mathbf{O}\left(x, y, \lambda_{1}\right) \text {, and } \mathbf{X}\left(x \frac{\lambda_{2}}{\lambda_{1}}, \mathrm{y} \frac{\lambda_{2}}{\lambda_{1}} \lambda_{1}\right) .
$$

The two letters on the left are blue, and those on the right are green.

The polychromatic illumination is obtained from an argon ion laser using a broadband end mirror. By operating the laser at fairly low power levels, only the $\lambda_{1}$ and $\lambda_{2}$ wavelengths lase. In addition, adjustment of the laser current varies the ratio of the intensities of $\lambda_{1}$ and $\lambda_{2}$. This ratio is set to produce equal intensity images of the central $\mathbf{O}$ and $\mathbf{X}$ in the impulse response (Fig. 3) in our vidicon camera. In this way, compensation is made for slight differences in diffraction efficiency of the two superimposed MSFs and for the wavelength dependent response of the vidicon.

In Figs. 4 and 5, we show the results of using the color-coded MSF for optical correlation. The photograph in Fig. 4 shows the correlation plane output obtained when the object $\mathbf{O}$ is inserted in the input plane. The correlation signals $U_{31} \rightarrow U_{34}$ (from Table II) and output wavelengths are identified from left to right below the photograph. The plot above the photograph is obtained by placing a vidicon in the correlation plane, digitizing the output image, and plotting the intensity along a line passing through the centers of all the correlation peaks.

The green autocorrelation of the $\mathbf{O}$ (third term from left) is the most intense signal. The blue XO cross correlation (second term) is much weaker. These two terms would be superimposed if the MSF were constructed with $x_{0}=0$, but the intense green correlation peak would identify the input object as the $\mathbf{O}$. Note that the cross correlation between the scale-changed $\mathbf{O}$

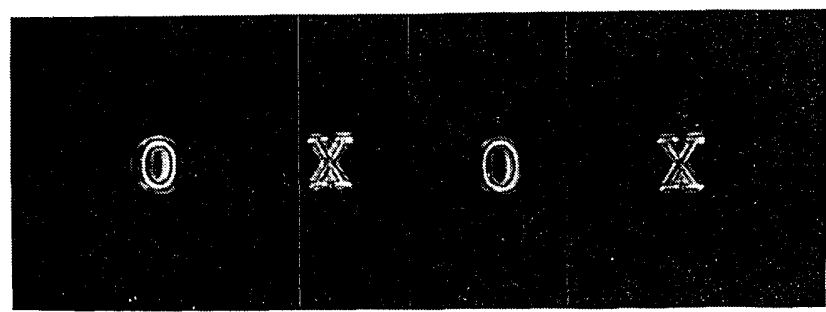

Fig. 3. Impulse response of double-exposure matched spatial filter.

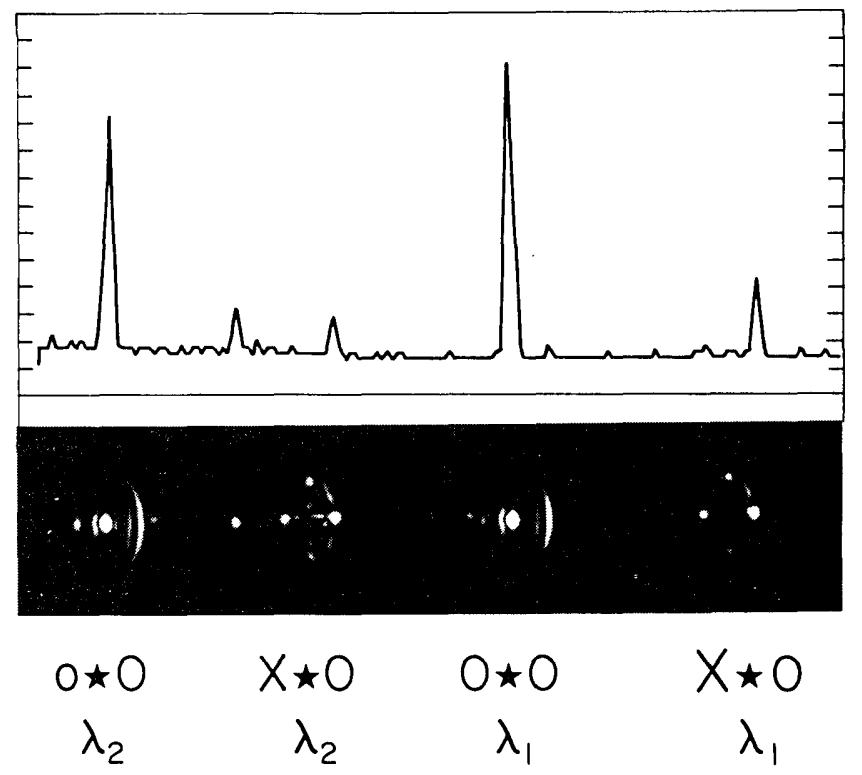

Fig. 4. Correlation plane output with $\mathbf{O}$ input.

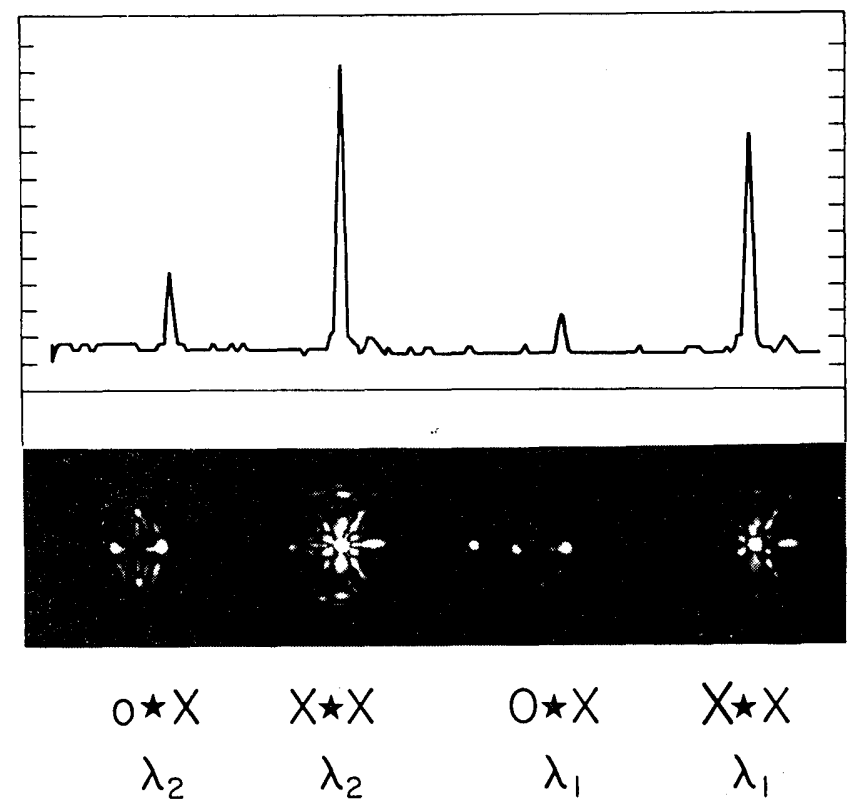

Fig. 5. Correlation plane output with $\mathbf{X}$ input.

and the input $\mathbf{O}$ (first term) is still quite intense because the size change is only $5 \%$. This term can always be spatially separated from the two center terms.

The photograph in Fig. 5 shows the correlation plane output (terms $U_{35} \rightarrow U_{38}$ from left to right) obtained when the object $\mathbf{X}$ is inserted in the input plane. The plot shows that the blue $X$ autocorrelation (second term) is now much more intense than the green $\mathbf{O X}$ cross correlation (third term). Thus the intense blue signal indicates that the $\mathbf{X}$ is present at the input. 


\section{Discussion}

We have shown that color-coding or wavelengthmultiplexing of several matched spatial filters onto one piece of film can be used for pattern recognition operations. While wavelength-multiplexed filters are more difficult to construct than a series of single exposure filters, they are easier to use in that they require no sequential positioning. This can lead to cost, weight, and space savings in critical applications. Wavelengthmultiplexed filters also allow parallel recognition operations where a single color-discriminating detector can be used at the output of an optical correlator for the simultaneous recognition of several objects. In addition, the color-coded correlation signals can serve as visual cues to human observers.

Many practical systems might require just a few superimposed filters so that conventional recording materials could be used. The maximum number of filters realizable on one piece of film depends on the recording geometry and film type. For reflection filters recorded with 120 - $\AA$ reflection bandwidth, ${ }^{8}$ twenty-five filters could be recorded within the visible spectrum. Of course, bias buildup problems from this number of exposures in conventional recording materials would cause considerable difficulty. ${ }^{9}$ However, for phase materials such as dichromated gelatin, ${ }^{10}$ which can achieve very large index modulations or new materials such as porphins, ${ }^{11,12}$ which have a very narrow wavelength response, a large number of MSFs could be superimposed.

Although only two-wavelength readout was used for these experiments, broadband (white light) illumination could also be used. In an off-axis transmission system, the additional cross-correlation signals present because of the additional wavelengths will be spatially displaced from and of lower normalized intensity than the autocorrelation signal. Thus, the autocorrelation signal could be separated from undesired signals.

The decrease in signal/noise experienced when many filters are conventionally multiplexed ${ }^{13}$ could be avoided with this technique because the autocorrelation signal and the noise signals at a given output location are of different wavelengths and could be spectrally isolated.
The'author thanks Jean-Marc Fournier and Bruce Hansche for useful discussions. Silverio Almeida and Judy Partin are also acknowledged for their assistance. This work was partially supported by NSF Grant No. ENV77-10184.

Note added in proof: The reader is referred to additional recent articles on color-coded pattern recognition. $\mathrm{Shi}^{15}$ has calculated the wavelength selectivity of reflection matched spatial filters. In addition, Bartelt ${ }^{16}$ has demonstrated a white light, image plane correlator which produces color-coded correlation signals.

\section{References}

1. J. Bescos and T. C. Strand, Appl. Opt. 17, 2524 (1978).

2. J. D. Armitage, A. W. Lohmann, and D. P. Paris, Jpn. J. Appl. Phys. 4 (Suppl. 1), 273 (1965).

3. A. A. Friesem and U. Levy, Opt. Lett. 2, 133 (1978).

4. A. Vander Lugt, F. B. Rotz, and A. Klooster, in Optical and Electro-Optical Information Processing, J. T. Tippett et al., Eds. (MIT Press, Cambridge, 1965).

5. S. P. Almeida and J. K. T. Eu, Appl. Opt. 15, 510 (1976).

6. Recording several superimposed filters, each at the same wavelength but with a different reference beam angle, would also reduce filter positioning requirements.

7. J. W. Goodman, Introduction to Fourier Optics (McGraw-Hill, New York, 1968), p. 176.

8. R. J. Collier, C. B. Burckhardt, and L. H. Lin, Optical Holography (Academic, New York, 1971), p. 258.

9. H. Fleisher et al., in Optical and Electro-Optical Information Processing, J. T. Tippett et al., Eds. (MIT Press, Cambridge, 1965), p. 1.

10. S. K. Case and R. Alferness, Appl. Phys. 10, 41 (1976).

11. B. Braunecker, University of Essen, FB Physik, 4300 Essen 1, West Germany; private communication.

12. S. Voelker, R. M. MacFarlane, and J. H. Van Der Waals, Chem. Phys. Lett. 53, 8 (1978).

13. See, for instance, R. G. Zech, "Data Storage in Volume Holograms," Thesis, University of Michigan (1974) (University Microfilms, Inc., order 74-25, 369), p. 257.

14. The system could also be used in reverse for code translation. A green point source (such as a $\lambda$-multiplexed signal emerging from a fiber optic) could be used to produce the word GO, while a red point signal would produce the word STOP.

15. N. K. Shi, Opt. Lett. 3, 85 (1978).

16. H. Bartelt, Opt. Commun..(May 1979), accepted for publication.

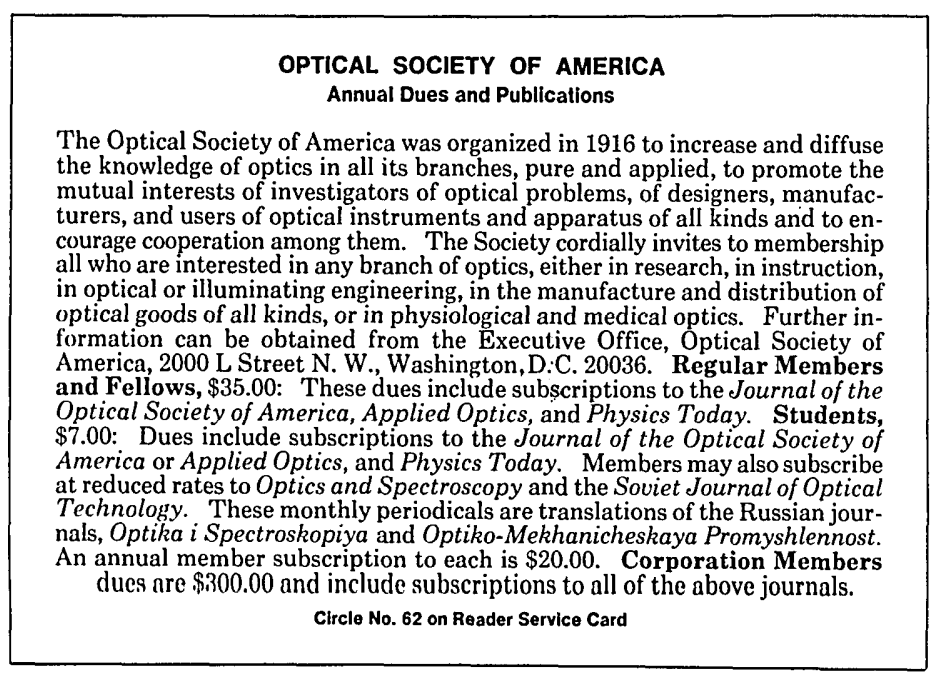

